\title{
In-situ TEM Heating Experiment to Study Effects of Cyclic Thermal Gradients on Ti-6Al-4V under Additive Manufacturing Growth Conditions
}

\author{
Meiyue Shao ${ }^{1}$, Cheng-Han Li $^{1}$, Curtis L. Frederick ${ }^{2}$, Sudarsanam S. Babu ${ }^{2,3,4}$ and Joerg R. Jinschek ${ }^{1}$ \\ 1. Department of Material Science and Engineering, The Ohio State University, Columbus, $\mathrm{OH}$. \\ 2. Department of Material Science and Engineering, University of Tennessee, Knoxville, TN. \\ 3. Department of Mechanical Aerospace and Biomedical Engineering, University of Tennessee, \\ Knoxville, TN. \\ 4. Advanced Manufacturing Program, Energy and Environmental Sciences Division, Oak Ridge National \\ Laboratory, Knoxville, TN.
}

Additive manufacturing (AM) [1] is a layer by layer manufacturing process, which is increasingly applied to produce parts and prototypes in automotive, aircraft and aerospace industry, as well as implants and prosthesis in biomechanical industry. In the AM process, a metal powder with targeted metal alloy composition is exposed to a scanning laser or electron beam, thereby experiencing a cyclic thermal process with large thermal gradients in rapid heating-cooling cycles. As an example, Ti-6Al-4V powder (AM Ti64) has been commercialized for laser-melting AM process [2] based on its special characteristics of a low weight ratio, as well as outstanding corrosion resistance, attractive for weight-reduction technology in transportation vehicles. However, in order to eventually replace established technology, it is necessary to understand properties of AM-produced parts based on the relationship between microstructure and processing parameters. Kenel.et [3] investigated phase transformation in AM Ti64 under laser melting and rapid cooling cycle by high-speed micro-X-ray diffraction (XRD) and $\alpha^{\prime} \leftrightarrow \beta$ phase transformation was observed repeatedly.

SEM-EDS mapping on pristine AM Ti64 (see Fig.1a) shows an average particle size of 30-40 $\mu \mathrm{m}$ and confirms the average chemical composition [2]. SEM contrast changes on the sphere of the particle indicate presence of grain boundaries. Using focused ion beam (FIB) techniques, electron transparent lamellas have been extracted from these areas for further TEM investigations (see Fig.1b and 1c).

TEM experiments (including in-situ heating) and STEM-EDS characterizations (Fig.2) are performed. The Bright Field TEM image (Fig.2a) and diffraction pattern on [0001] zone axis (Fig.2b) confirm the hexagonal-closed-packed (HCP) $\alpha$ phase of AM Ti64 at room temperature. STEM-EDS spectrum (Fig.2d) shows a uniform chemical composition.

In-situ TEM heating experiment provide access to structure and dynamic behavior under AM processing conditions on the nanometer scale. MEMS-based heating stages with fast thermal response (up to $10,000 \mathrm{~K} / \mathrm{sec}$ ) and accurate temperature measurement [4] offer a powerful tool to monitor microstructure evolution during fast heating and cooling process. Here, we use (in situ) S/TEM methods to observe phase transformations in AM Ti64.

During the heating cycle at $990^{\circ} \mathrm{C}$, primary $\mathrm{HCP} \alpha$ phase transfers to body-centered-cubic (BCC) $\beta$ phase. And in the subsequent cooling cycle, BCC $\beta$ phase transfers to hexagonal $\alpha$ ' martensite. STEM-EDS mapping across the grain boundaries will be used to understand if diffusion phenomena cause differences in elemental distribution. Finally, atomic scale S/TEM characterization will be conducted to observe dynamic changes and to understand solid/solid phase evolution and grain orientation in fast heating- 
cooling cycles [5].

References:

[1] W.E. Frazier, Journal of Materials Engineering and Performance 23 (2014), p. 1917.

[2] http://www.arcam.com/technology/products/metal-powders/

[3] C. Kenel et al, Scientific Report 7 (2017), p. 16358.

[4] L. Mele et al, Microscopy Research and Technique 79 (2016), p. 239.

[5] The authors acknowledge funding from Ohio State's Institute of Materials Research (IMR) Facility Grant (IMR-FG0209).
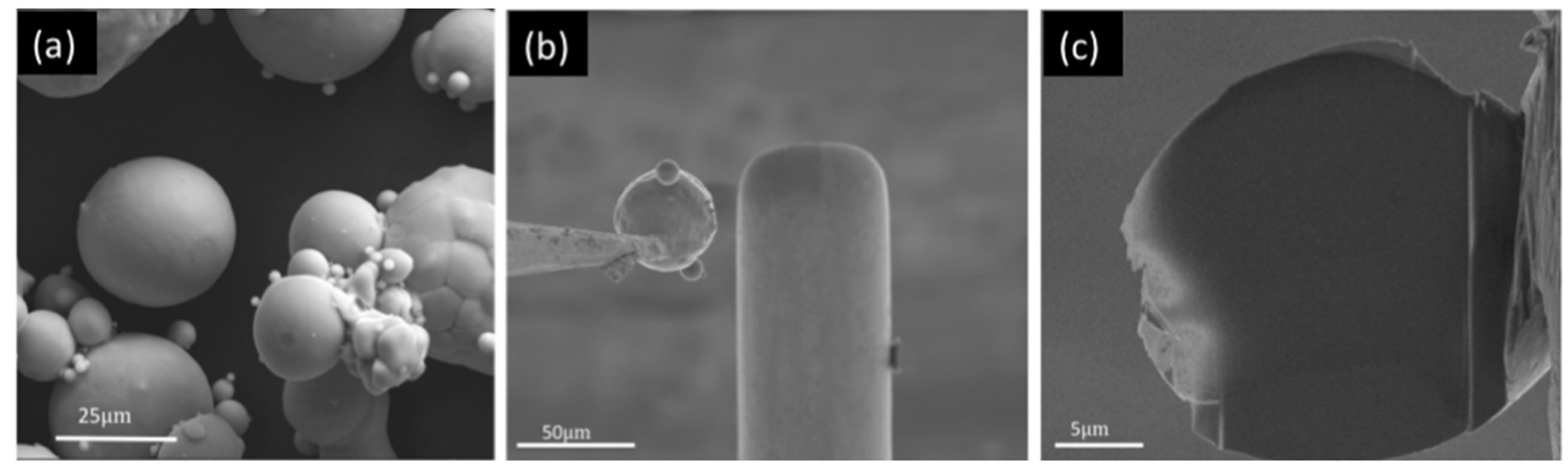

Figure 1. (a) SEM image of AM Ti64 powder particles; (b) one AM Ti64 particle attached to Omniprobe to transfer to a TEM grid; (c) the final electron transparent lamella prepared by FIB milling.
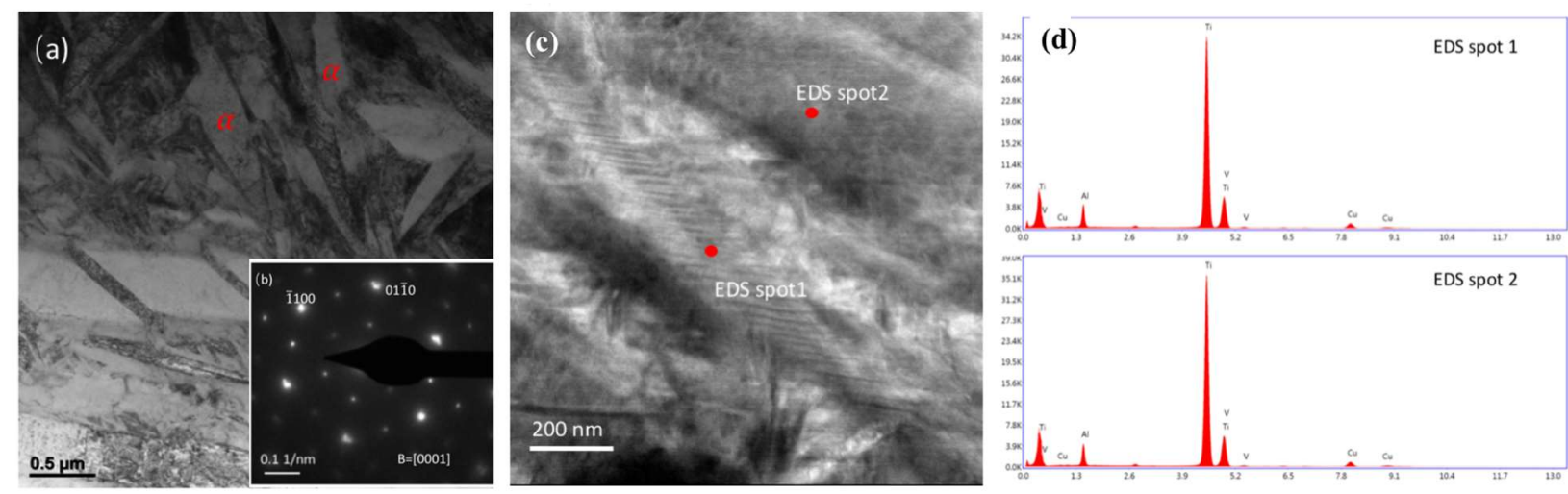

Figure 2. (a) Bright field TEM image and (b) one diffraction pattern showing [0001] zone of HCP $\alpha$ phase; (c) STEM image of Ti64 and (d) the STEM-EDS spectrum of spots marked in (c) 